\title{
Novel Photoflexoelectric Membranes
}

\section{A.G. Petrov from the Bulgarian Academy of Sciences, Sofia, describes possible molecular elec- tronic devices based on photoflexoelectricity, a novel effect in specially designed membranes that combines the response to light, curvature and electric potential.}

Flexoelectricity - the liquid-crystal analogue of piezoelectricity - is a fundamental property of liquid crystals that relates their mechanical and electrical degrees of freedom [1]. It also arises in liquid crystalline biostructures, i.e., in biomembranes [2]. In membranes, both living and model ones, flexoelectricity provides a direct as well as an inverse linear relationship between membrane curvature and membrane polarization $[2,3]$. The phenomenon is closely related to mechanosensitivity and mechanotransduction, basic features of living systems [4]. Understanding membrane mechanosensitivity would open the way towards constructing bioelectronic mechanosensors and actuators having mechanical input and electrical output, or vice versa.

Until recently, however, introducing an additional optical degree of freedom to membrane systems remained relatively unexplored in the context of flexoelectricity. The interest in including the optical degree of freedom seemed obvious: an opto-mechano-electric membrane model system would greatly increase the number of possible combinations between the three degrees of freedom (either inputs or outputs). While applications in molecular electronics, ionics and photonics could be very interesting, the demonstration of the existence of all three degrees of freedom in some native membranes would, above all, lead to new hints about the relationship between structure and function in photoactive membranes.

Our group in Sofia made some early observations of an illuminationdependent flexoelectric response - an effect we call "photoflexoelectricity" - in 1983 using bilayer lipid membranes (BLM) containing the photoactive protein bacteriorodopsin, and in 1992 using photoactive molecules of retinal acetate. We recently

Alexander Petrov is Professor of Physics in the University of Sofia and Head of the Biomolecular Layers Department, Institute of Solid-State Physics, Bulgarian Academy of Sciences, 72 Tzarigradsko chousse, BG-1784 Sofia. He received a PhD from the Academy in 1974 and has worked at Nottingham University, Syracuse University (NY, USA) and the Université de Paris-Sud. The research reported here was carried out under the Bulgarian - US National Academy of Sciences cooperation that was launched in the early-1990s. reported observations of the same response for BLM coated in situ with quantum-sized semiconductor particles [5]. The phenomenon can in a certain sense be regarded as a liquid-crystal analogue of photo-piezoelectricity which is observed in some solid crystals.

The experiment was as follows: the flexoelectric response of glyceryl monooleate (GMO) bilayer lipid membranes, both bare and decorated by photosemiconductor CdS nanocrystallites, was measured in the dark and under bandgap illumination by simultaneously applying an oscillating pressure to one side of the BLM that varied in the $100-1000 \mathrm{~Hz}$ frequency range. Electric currents generated under a fixed voltage and membrane potentials generated under a fixed current were measured using a phase-sensitive detection technique. The bare GMO membrane gave an illumination-independent flexoresponse, as did all other non-photoactive lipid membranes studied so far. On the other hand, a reversible increase of the flexoelectric response of the CdS-

decorated GMO BLM during illumination (i.e., photoflexoelectricity) was observed.

The curvature-induced root-mean squared membrane potential (at zero fixed current) during light illumination and during successive dark periods is plotted in the figure. It can be seen that the changes in the amplitude of the response correlated with the light and dark states of the membrane. In particular, an increase of the flexoelec- tric response after a flash of light was followed by a return to the initial dark stage after the light was switched off: simultaneous jumps in the relative phase were also observed. The photoflexoelectricity varied between $3-100 \%$ of the dark response depending on the amount of CdS deposited on the BLM, and the amplitude and frequency dependence were measured.

The time evolution of the photoflexoelectric response after initiation of the particle growth can be understood on the basis of the previously studied time dependence of the photopotential, modulated by the BLM's curvature oscillations.

The development of our model system was prompted by its mimicking photoelectron transfer in photosystems based on naturally occurring membranes. The system combines two states of matter namely, a liquid crystalline lipid membrane and solid crystalline (proteinmimicking) nanoparticles. The observed relationship between the membrane shape (curvature), light intensity and the membrane potential can be related to the mechanism responsible for phototaxis at the cell membrane level, and the possibility of observing the inverse effect, i.e., lightinduced curvature, would be of a special interest in this respect [3]. Several concepts for mechano-electro-optical sensors and actuators for molecular electronics utilizing this new effect are presently under development.

\section{References}

[1] R.B. Meyer, Phys. Rev. Lett. 22 (1969) 918. [2] Petrov \& Derzhanski, J.Physique Suppl. 37 (1976) 155.

[3] Todorov, Petrov \& Fendler, Langmuir 10 (1994) 2344; J. Phys. Chem. 98 (1994) 3076. [4] Petrov \& Usherwood, Eur. Biophys. J. 23 (1994) 1.

[5] Spassova, Petrov \& Fendler, J. Phys. Chem. 99 (1995) 9485.
Flexoelectric response at a fixed zero current of CdS-decorated glyceryl monooleate (GMO) bilayer lipid membrane (BLM) at a pressure-induced oscillation frequency of $700 \mathrm{~Hz}$. The first harmonic of the membrane potential is plotted. The increase of the flexoelectric signal after a flash of light is reversible and shows exponential kinetics. The CdS nanosized particles were generated in situ by adding $\mathrm{CdCl}_{2}$ to the $0.1 \mathrm{M} \mathrm{KCl}$ electrolyte in contact with one side of the membrane, and by injecting $\mathrm{H}_{2} \mathrm{~S}$ gas into the same electrolyte in contact with the other side of the membrane. Growth of the CdS crystallites on the GMO BLM was monitored by optical microscopy.

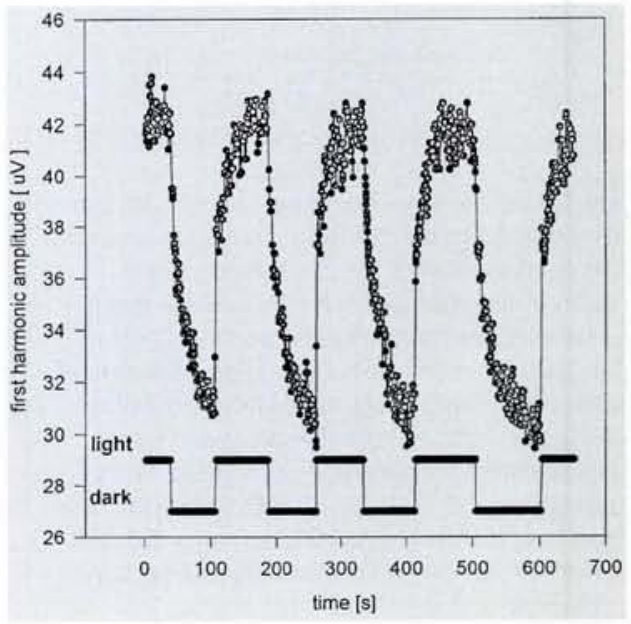

